\title{
Arts As A Possibility Of Health Promotion: A Proposal For Primary Care
}

\author{
Lavinia Teixeira-Machado \\ PhD Education in Health Department. \\ Federal University of Sergipe, Brazil. \\ Eduardo Tessari Coutinho \\ PhD School of Performing Arts. \\ University of São Paulo, Brazil.
}

\begin{abstract}
This manuscript discuss the arts as a possible and effective intervention in primary health care. The aim is to highlight health care and promotion policies in Brazil and how education and lifestyle modification can be accomplished. Arts in health promotion and well-being enable and influence the overcoming of the precariousness and inequity of health actions. Primary Health Care is the most efficient way of organizing health system. In the sphere of health promotion policy, community participation stands out as essential to ensure its effectiveness. Health education through arts generates an interface with culture, being fundamental for the promotion of conditions that improve daily activities. Edification of health education ways, using arts, fosters a new vehicle for expression, interpretation, representation and life style. Arts socialize information inherent in various artistic forms, by bringing popular knowledge closer to educational success. As value is conferred on lived experience, art meaning is essentially linked to health. Arts enhance verbal and non-verbal communication, flooded with meanings and symbolism, there are interferences in feelings, in the way people perceive themselves and perceive the world around them, and consequently how they shape their health and their being in the society in which they live.
\end{abstract}

Keywords: Arts, Primary Health Care, Health Promotion, Popular Knowledge

"We have Art so we will not die of the truth". Friedrich Nietzsche

\section{BRIEF PRELUDE}

Thinking about arts in health promotion and well-being enables and influences the overcoming of the precariousness and inequity of health actions, with a view to acting in the collective and transforming lifestyle, which is substantial for the promotion of autonomy and empowerment of community.

Although we perceive the precariousness of public health in Brazil, which reinforces deficiency in the entire care network, we can not neglect the exponential jump in Brazilian public policy through the formulation of the Federal Constitution at 1988, with the Unified Public Health System (SUS - Sistema Único de Saúde) creation, although services still go a long way to becoming satisfactory $[1,2]$.

Incessant search for adequate health and life conditions feed into the creation and implementation of feasible alternatives, from the reformulation and organization of paradigms and principles. Analysis of the use of the arts in the health services, awakens an aspect of health practice: the prevention-cure-rehabilitation process, based on the professional duality 
of health and the user, participating together and in a participatory way, in order to effectively transform results, modifying the scenario and deconstructing paradigms in the course of health construction [3-5].

Technological insufficiency, lack of maintenance and equipment scrapping, negligible number of specialized professionals, difficulty of users' seizure of information, among other shortcomings in the SUS, generate frustrations and subsequent dehumanization in the service.

However, it would be audacious to elucidate that arts have the peculiarity of filling these gaps, but starting from the presupposition of symbolism, and having art as a means of representing symbols: according to Hospers [6], the way of embracing ideas of separation and union, for evoking a uniformity that has been divided and regroupable, revealing itself as representation and not as reproduction.

Art can be expressed, interpreted, represented, experienced. It stimulates different parts of the brain and the central nervous system itself - incites emotions; in a dynamic search between feel-think-act, being a key element for the promotion of quality of life and health [7-9].

This is because arts promote complex interactions between body-environment, through the perception of sensations that allows the possibility of self-organization, self-confidence and, above all, well-being [10-12].

In the conjectures of Kazi \& Peter [13] and Tan \& Davis [14], they argue the claim of Theodor Billroth, known as the father of modern abdominal surgery and also talented instrumentalist, the following thought: "is one of the superficialities of our time judge science and art as opposing. Imagination is the mother of both."

Arts promote the interaction between knowledge and practice that are interrelated with the history and culture of societies, allowing the teaching / learning process in an effective way, through experiences lived in different spheres [4, 15-16].

Since arts contain a universe of pedagogical components of great capacity to transform reality. In addition to the peculiarity of creating links: a substantial process for social reconstruction, in the sense of providing reintegration of the individual to the scenario in which he is inserted as protagonist actor.

\section{Primary Health Care in Brazil}

Basic Health Care (BHC) is based on the construction of links for the promotion of the health of all Brazilians. BHC encompasses a set of values and sedimented precepts in the principles and guidelines that make up the SUS.

Primary Health Care (PHC) in other countries (BHC in Brazil), is the most efficient way of organizing health system. International evidence shows that health-oriented PHC systems have shown benefits, lower costs, and more satisfactory outcomes.

According to the Ministry of Health in Brazil, BHC is

"a set of actions, of individual or collective character, located in the first level of attention of the health systems, aimed at health promotion, prevention of diseases, treatment and rehabilitation [...]" [1]. 
Starfield, $\mathrm{Xu}$ and Shi [17] elucidate four essential parameters for health services in PHC: access of the user's first contact, longitudinality, completeness and coordination of care. In addition, three derived characteristics qualify the actions of services to the health: attention to the health centered in the family, community orientation and cultural competence [17-18].

Various propositions of arts as a course of communication and education have as pillars culture and diversity, considering socio-cultural inclusion, manifestation of skills and competences in the scope of art and health, strategies of transformation of behaviors that aim at changing attitudes and behaviors, promoting human development and social responsibility $[10,15,19]$.

Some common attributions of health professionals in the SUS are: "to ensure the integrality of care through health promotion actions, prevention of injuries and curatives, and assurance of spontaneous demand, programmatic and surveillance; promoting community mobilization and participation, seeking to achieve social control; participating in continuing education activities; carry out other actions and activities in accordance with local priorities " [1].

\section{HEALTH PROMOTION}

Health Promotion is a structuring axis of the SUS as a policy, which aims at an expanded, complex and integrated perception about the concept of health and life, being multifaceted and intersectoral, as it articulates with the environment, education, human resources, life style and quality of life [2].

Groundwork for the real guarantee of health promotion is based on expansion of the concept of health, a substantial resource for personal, biological, economic and social progress that transcends health sector, with fundamental requirements being the guarantee of well being, education, housing, food, income, sustainable ecosystem, social justice and equity [20-21].

In the sphere of health promotion policy, community participation stands out as essential to ensure its effectiveness. Services should be organized through strategies that enable health promotion actions, as well as attitudinal and behavioral changes of health professionals focused on the needs of the social actor inserted in his environment.

\section{ARTS AND HEALTH PROMOTION}

Arts positively influence health promotion, as they act on fundamental mechanisms of learning and understanding of the human being, whether he is suffering from some kind of health problem or not. Approximation of arts to health education facilitates apprehension, participation and cultural practice $[9,19,22]$.

This is because art configures the link of the real and the symbolic through complexity, contemplation and perplexity, which predispose the multifaceted perception that it fosters. Body-environment acts as a symbolic entity that is real, presenting infinite details, often imperceptible to some, but interfering in the collective unconscious for symbolic structuring of the construction and deconstruction of the world as revelation $[3,8,12]$.

Education in health course is an essential attribution of caring work in the care networks, supported in the dialogue between people (health professionals and population) with the objective of mobilizing forces and motivating changes (behavioral, attitudinal or adaptations) to new life situations [7,23-24]. 
Emotional health fostered by arts is capable of regressing clinical conditions of a progressive and irreversible conditions, probably by stimulating dormant sensations that reinvigorate and mobilize creativity and social integration, fundamental factors for the human being to feel part of a whole $[4,19]$.

This subjective condition reflects on well-being, precisely for evoking continuing education, from the development and implementation of pedagogical practices in health, in the sense of strengthening humanization and health promotion advocacies, providing a more comprehensive vision and the dialogue between several fields of knowledge $[9,23]$.

Health education through arts generates an interface with culture, being fundamental for the promotion of conditions that improve daily activities. Performing arts can be seen as a dramatic educational game that reaches human being in its entirety, encompassing creativity and learning through playfull activities $[8,11]$.

It is possible to elucidate that dramatic art, based on representations of conjunctures of moments, situations and problems, involves collective social practice, regardless of whether or not it is part of performance. Use of music, critical theatrical language, and art education impel natural educational process, subsidized in understanding and transformation of reality to which social actor is inserted $[3,8,16]$.

On the other hand, music and dance trigger emotional states that generate well-being, selfknowledge and reduction of depression and resignation, because it dialogues through bodily awareness, fundamental for normalization of compensatory mechanisms essential for bodily stability. And the moment one becomes aware of the deeper causes of health problems and the social relations that permeate it, intervention, in turn, becomes conscientious about misfortunes caused by injuries and carelessness with health [4,6,19].

This fact owes to the peculiarity of arts to impart passion, breaking settled internal balance, and modifying the will to feel new, by awakening dormant emotions essential for transcendence of deleterious vices. Art brings the personal who reflect on the social, as it illustrates the potential vehicle of awareness through intentionality and reciprocity, both involved in meaning and excellence. In addition, playful activity contemplates fundamental criteria for effective learning, configuring and transporting itself to the reality.

\section{FINAL CONSIDERATIONS}

As explained by Vygostsky (1896-1924), "Art is the social in us," a reflection of fundamental importance for aesthetic-ontological knowledge, in the sense of seeing art as an essential role in the propagation of new models and lifestyles, for the formation and preparation of new consciences based on life adequate to the environment [25].

Art is emphasized in social policies, by the diversification of new forms of health actions, as processes of identity construction and emancipation through symbolization of the dialectical thinking of the impact on personal life, without refuting social being, since it makes possible to re-signify not only as health services are organized, but proposes to review and interpret the concept of health in its broadest sense as proposed by health promotion policy.

Edification of health education ways, using arts, fosters a new vehicle for expression, interpretation, representation and life style. Arts socialize information inherent in various artistic forms, by bringing popular knowledge closer to educational success. 
For example, the use of lecture only, as it has been widely used in health education, is insufficient to achieve success in transmission of learning and habit change. By this way, arts have power to increase apprentice's capacity for absorption of information due to the fact that arts penetrate the core of the human being not only by the visual and auditory systems, but by influencing all sensory systems, that is, is an organic educational form.

As value is conferred on lived experience, art meaning is essentially linked to health, it inspires salutary mind conception that propagates the energy transmitted by artistic manifestation in various prisms. Arts enhance verbal and non-verbal communication, flooded with meanings and symbolism, there are interferences in feelings, in the way people perceive themselves and perceive the world around them, and consequently how they shape their health and their being in the society in which they live.

\section{References}

Brasil (2006) Health Ministry. Ministerial order number 648, March 28, 2006. National Policy of Basic Attention in Health.

Brasil (2010) Health Ministry. Primary Care Assessment Tool, PCATool-Brasil. Brasília, 2010. 80p.

Cohen G (2009) New theories and research findings on the positive influence of music and art on health with ageing. Arts \& Health: An International Journal for Research, Policy and Practice, 1(1):48-62.

Purser A (2017) Dancing Intercorporeality: a health humanities perspective on dance as a healing art. J Med Humanity, doi: 10.1007/s10912-017-9502-0.

Teixeira-Machado L, DeSantana JM (2015) Dance Therapy improves quality of life in individuals with neuromotor disorders: randomized controlled trial. International Journal of Humanities Social Sciences and Education, 2(4):84-92.

Hospers J (1948) Meaning and Truth in the Arts. The Journal of Aesthetics and Art Criticism, 6(4):352-355.

Bat-Or M, Garti D (2018) Art Therapist's perceptions of the role of the art medium in the treatment of bereaved clientes in art therapy. Death Stud. doi: 10.1080/07481187.2018.1445138.

Keightley M, Agnihotri S, Subramaniapillai S, Gray J, Keresztesi J, Colantonio A, Polatajko HJ, Cameron D, WisemanHakes C (2018) Investigating a theatre-based intervention for indigenous youth with fetal alcohol spectrum disorder. Can J Occup Ther. 2018 Jan 1:8417417719722.

Mojarrab S, Rafei A, Akhondzadeh S, Jeddian A, Jafarpour M, Zendehdel K (2017) Diseases and Health outcomes registry systems in I.R. Iran: sucessfull initiative to improve public health programs, quality of care, and biomedical research. Arch Iran Med, 20(11):696-703.

Osborne J (2010) Art and the child with autism: therapy or education? Early Child Development and Care Journal, 173(4):411-423.

Slayton SC, D'Archer J, Kaplan F (2011) Outcome studies on the efficacy of Art Therapy: a review of fidings. Art Therapy Journal, 27(3):108-118.

Varela F, Thompson E, Rosch E (1993) Embodied mind: cognitive science and human experience. Londres: The MIT Press. 1993, 295p.

Kazi RA, Peter RE (2004) Christian Albert Theodor Billroth: master of surgery. J Postgrad Med, 50:82-83.

Tan SY, Davis CA (2008) Theodor Billroth (1829-1894): pioneer of modern surgery. Singapore Med J, 49(1):4.

Lusebrink VB (2011) Art therapy and the brain: an attempt to understand the underlying processes of art expression in therapy. Art Therapy Journal, 21(3):125-135.

Tanaka S (2015) Intercorporeality as a theory of social cognition. Theory Psychol, 25(4):455-472.

Starfield B, Xu J, Shi L (2001) Validating the Adult Primary Care Assessment Tool. The Journal of Family Practice, 50(2):161-175.

Starfield B (1994) Is primary care essential?, 344:1129-1133.

Teixeira-Machado L, Azevedo-Santos I, DeSantana JM (2017) Dance improves functionality and psychosocial adjustment in cerebral palsy: a randomized controlled clinical trial. Am J Phys Med Rehabil, 96(6):424-429. 
Boscarelli A (2017) Clown therapy: not only a pediatric matter. Transl Pediatr, 6(2):111-112.

Leguizamon RC, Castaño OA, Morales GC, Cifuentes NL (2017) Humour therapy intervention to reduce stress and anxiety in paediatric anaesthetic induction, a pilot study. Br J Anaesth, 119(4):847-848.

Tanaka S (2017) Intercorporeality and aida: developing an interaction theory of social cognition. Theory Psychol, 27(3):337-353.

Whitehead PM (2015) Overcoming parallelism: Naturalizing phenomenology with Goldstein and Merleau-Ponty. Prog Biophys Mol Biol;119(3):502-9.

Wagemann J (2018) The confluence of perceiving and thinking in consciousness phenomenology. Front Psychol, 8:2313.

Derouesné C (2017) Lev Semenovich Vygotsky (1896-1924): an introduction to the historico-cultural psychology and the development of the higher psychological processes. Geriatr Psychol Neuropsychiatr Vieil, 15(3):295-307. 